\title{
Género y enfoque historico-social. Las mujeres en el tiempo
}

\author{
Gênero e abordagem histórico-social: as mulheres no tempo \\ Gender and the socio-historical approach: women throughout \\ time
}

\section{Rosa Falcone*}

Universidad de Buenos Aires - UBA, Buenos Aires, Argentina

\begin{abstract}
RESUMEN
El presente artículo se propone sistematizar desde una perspectiva histórica los debates más significativos que promuevan, desde una posición crítica, la reflexión sobre lo femenino-masculino. En el marco de nuestra investigación se postula el enfoque histórico-social, como uno de los modos de comprensión de los procesos de producción de la subjetividad de género y de abordaje de lo femenino, a fin de contribuir a los debates actuales sobre la diversidad sexual, los feminismos y los estudios del género en general.
\end{abstract}

Palabras Clave: Psicología, Historia, Argentina, Mujeres, Feminismos.

\section{RESUMO}

O presente artigo propõe sistematizar, a partir de uma perspectiva histórica, os debates mais significativos que promovem, desde uma posição crítica, a reflexão sobre o feminino-masculino. No marco de nossa pesquisa, a abordagem histórico-social é postulada como uma das formas de entender os processos de produção da subjetividade de gênero e de abordagem do feminino, a fim de contribuir para os debates atuais sobre a diversidade sexual, os feminismos e os estudos de gênero em geral.

Palavras-chave: Psicologia, História, Argentina, Mulheres, Feminismos.

\begin{abstract}
The present article proposes to systematize, from a historical perspective, the most significant debates that promote, from a critical position, the reflection on the feminine-masculine. Within the framework of our research project, the socio-historical approach is proposed as the way to understand the process of gender subjectivity production and to approach the feminine, in order to contribute to the currents debates on sexual diversity, feminisms, and gender studies in general.
\end{abstract}

Keywords: Psychology, History, Argentina, Women, Feminisms. 


\section{NTRODUCCI ÓN}

En el marco de la investigación en curso, el presente artículo se propone sistematizar los últimos resultados obtenidos de la aplicación del enfoque histórico-social, al análisis de las diversas problemáticas de género ${ }^{1}$. Al respecto se ha planteado la necesidad de definir períodos históricos, con el propósito de plantear un seguimiento que muestre la inserción social de las mujeres y sus roles de acuerdo al contexto y efectuando estudios comparativos. De modo que, en continuidad con investigaciones previas, se propone en esta oportunidad, analizar el grado de incidencia de las transformaciones políticas, sociales y económicas en los procesos de construcción de la subjetividad de género. Nuestra premisa considera que dichas transformaciones, producidas en Argentina, en el transcurso de la primera mitad del siglo XX, han contribuido a la construcción de la subjetividad femenina, y que este análisis amplía la comprensión y abordaje de lo femenino en las sociedades contemporáneas.

Para arribar a dicho objetivo se privilegiará la mirada longitudinal y las metodologías que ponen el acento en las "tendencias" y "cambios de tendencias" (Cook \& Reichardt, 1986), tomando parcialmente de esta perspectiva, y como una construcción interesante, la idea de las "mujeres en el tiempo". La conjunción del análisis de las tendencias y la idea de las "mujeres en el tiempo" resultan metodológicamente apropiadas, no sólo porque revelan una evolución, sino porque permiten visualizar las variables que intervienen en los procesos de subjetivación de género, desde el marco teórico de las representaciones sociales. Se intentará demostrar, el modo en que las variaciones discursivas - producto de las transformaciones políticas, sociales y económicas- modifican la estructura del tejido social y repercuten en la familia y en las relaciones de género.

La importancia del método longitudinal, y de las transformaciones en el tiempo, es acentuada por varios autores al momento de reflexionar respecto de la incidencia de las variables sociales, políticas y económicas y el modo en que dichas variables intervienen en la comprensión del rol de la mujer a lo largo del tiempo: "La variable tiempo pasa a ser esencial para la validación del diseño de investigación y el diseño longitudinal buscará dar cuenta de la evolución prestando atención a las tendencias y cambios de tendencias en los fenómenos investigados" (Campbell \& Stanley, 1970).

El análisis del rol o papel de la mujer en el tiempo será comprendido en un anudamiento significativo entre los discursos jurídico, religioso, político e incluso el discurso médico-psiquiátrico, que, como intentaremos mostrar en el desarrollo de esta presentación, influyen en el cambio de tendencias. De este modo, el seguimiento de las formulaciones discursivas nos permitirá estudiar las representaciones 
de género, a partir del relevamiento parcial (ceñidos a la extensión del presente artículo), de los factores predominantes, que intervienen en la constitución de una sociedad, y en un momento determinado. Narvaja de Arnoux, desde sus investigaciones, refuerza el procedimiento de análisis de las formulaciones discursivas, a través del uso del análisis del intradiscurso y el interdiscurso en una complejidad interdependiente (Narvaja de Arnoux, 2006, 9-11). Van Dijk (1994) afirma del mismo modo que el interdiscurso, como conjunto estructurado e inestable de formaciones discursivas, ayuda en la delimitación y entrecruzamientos discursivos exponiendo una memoria ideológica-discursiva (Van Dijk, 1994).

Los aportes de Denise Jodelet, sobre el discurso y la representación como sistema de significación compartida, son útiles en la mirada longitudinal que estamos planteando. La autora sostiene que la representación busca elementos que son comunes a una unidad social y de ahí se puede recurrir a una organización de significados y contenidos como procesos simbólicos. Estas categorías resultan propicias para los análisis, a través del tiempo, en tanto tienen en cuenta las significaciones inherentes a los procesos sociales y los múltiples factores que intervienen en los procesos de construcción de la subjetivación. Asimismo, el fondo de la cuestión se apoya en las investigaciones de Castoriadis (1986), con su concepto de constitución imaginaria de la sociedad, cuyo conocimiento me exime de mayores desarrollos.

En consecuencia, en el marco de la importancia concedida a la teoría de las representaciones sociales de Jodelet y al imaginario social de Castoriadis, el presente artículo tiene el propósito de mostrar las transformaciones discursivas, que permiten comprender la evolución del rol femenino a lo largo del tiempo, comparando el lugar de la mujer "destinada al hogar" y el impacto de los cambios en la "mujer sostén del hogar" en las sociedades contemporáneas. Se intenta dar cuenta de la hipótesis que persigue nuestra investigación, que afirma la evolución del rol hogareño (doméstico) de la mujer hacia su participación en ámbitos públicos, y que dicha evolución va conformando su rol de género, aplicable a las sociedades contemporáneas, tal como veremos en las conclusiones.

El período de análisis para el presente estudio se circunscribe, entre 1900 y 1960, aproximadamente. Se trata de un momento de la historia argentina trabajado de diversos ángulos por distintos autores argentinos (Hugo Vezzetti, Lucía Rossi, Hugo Klappenbach, Ana María TalakAlejandro Dagfal ${ }^{2}$, entre otros) por considerar su importancia en la consolidación de las disciplinas psi (psicología, psiquiatría y psicoanálisis). La oportunidad del presente artículo, que se enmarca en una investigación más amplia, que tienen sede en varios proyectos 3 , es volver a estudiar este período de la historia argentina desde la perspectiva de género, por considerarse un momento relevante, que 
podría ayudar a comprender las relaciones de género en la actualidad.

\section{FUNDAMENTACIÓN TEÓRICO METODOLÓgICA: PERSPECTIVA DE GÉNERO}

El marco teórico de nuestra investigación apunta a ser original, en tanto plantea una mirada de género, en relación a un período de la historia argentina, de cuyo análisis se intentará extraer conclusiones respecto de los cambios en la representación social de las "mujeres en el tiempo". Nuestra perspectiva, que se refleja indirectamente en este artículo, vuelve sobre ideas formuladas por varios autores, y por nuestra propia producción en el área (Falcone, 2015), de las que se desprende que las significaciones de género nunca aparecen en forma pura, sino articuladas con otros aspectos determinantes de la subjetividad humana. Lo dicho presume, por un lado, que los roles, identidades $y$ valores atribuidos a varones $y$ mujeres son construcciones sociales e históricas; y por otro, que el género es una categoría de análisis pensada en términos relacionales ya que descubre las normas que determinan las relaciones entre mujeres y varones como una relación de poder. Citamos textualmente: “... porque no se refiere solamente a las relaciones entre los sexos, sino que alude también a otros procesos que se dan en una sociedad: instituciones, símbolos, identidades, sistemas económicos y políticos, etc."; y transversal "porque no están aisladas, sino que atraviesan todo el entramado social, articulándose con otros factores como la edad, estado civil, educación, etnia, clase social, etc." (Gamba, 2008).

Algunos historiadores advierten sobre el peso que tienen instituciones tales como la religión, los discursos científicos y los aparatos jurídicos. Pastor Ramos (1994), entre otros, sostiene que la raza, la religión y las clases sociales son algunos de los factores que se entrecruzan durante la constitución de nuestra subjetividad, y desde allí se podría hablar de procesos de subjetivación humana en el individuo singular. Scott afirma, en el mismo sentido, que cada cultura establece un conjunto de prácticas, discursos y representaciones sociales que atribuyen características específicas a mujeres y hombres (Scott, 1996, 1990).

En consecuencia y en este contexto, hacemos alusión al rol (papel) de género como una construcción. Suponer el carácter construido del papel de género implica ir contra la inevitabilidad atendiendo a lo que Ian Hacking enuncia como el punto de partida del construccionismo social: “(...) la existencia o el carácter de X no está determinado por la naturaleza de las cosas. $X$ no es inevitable, $X$ fue producido $O$ conformado por sucesos sociales, fuerzas, la historia, todos los cuales 
podrían perfectamente haber sido diferentes." (Hacking, 2001, p. 2627).

Decíamos también que la teorización de las relaciones de género se inscribe dentro de las relaciones de poder, caracterizadas por la desigualdad entre los sexos, y consideradas como una expresión más de la dominación social. En este sentido, nos apoyamos en los desarrollos teóricos de Bordieu (1983), cuando sostiene que los sujetos masculinos o femeninos son determinados mental $y$ corporalmente por medio de un orden simbólico, relacionado con la diferencia sexual, el que se manifiesta en jerarquías sociales. Este dominio simbólico se aloja y se reproduce en los esquemas prácticos del habitus, que complejiza la reflexión y por ende el cambio de conducta (Bordieu, 1983).Por ende, se postula el género como una construcción social, propia y diferente en cada cultura. El modo en que se estructura cada sociedad determina las relaciones de género y es desde esa interrelación que se instauran las bases fundacionales de las instituciones. Así creemos que se expresan las construcciones culturales, es decir, como creaciones sociales que exteriorizan los roles apropiados para hombres y mujeres y sus relaciones de poder.

Las investigaciones de Moscovici $(2000,1984)$ refuerzan la misma idea de tomar las representaciones sociales como sistemas de valores, ideas y prácticas, expresión de la sociedad y contribuyen a pensar que, por ser mecanismos necesarios para el establecimiento de identidades colectivas, tienen cierta continuidad en el tiempo. Esta función dinámica de la representación social, a diferencia de otras categorías usadas con frecuencia en psicología social (actitud, opinión), hace posible reconstruir las imágenes de una época.

Denise Jodelet, agrega al respecto, que hay un punto de intersección donde se funde lo individual con lo social: "hay sujetos sociales con una particularidad y una subjetividad propia que tiene que ver con las representaciones (...)" (Jodelet, 1986, p. 474). La representación social contiene implícitamente un significado, que lejos de ser una simple reproducción pasiva de un exterior en un interior, participa del imaginario social e individual y tiene relación con la situación del sujeto frente al mundo en que vive y con el que se relaciona, al tiempo y a la época (J odelet, 2007).

\section{LAS MUJ ERES EN EL TIEMPO DESDE EL ENFOQUE HISTÓRICO- SOCI AL}

Se propone esbozar en este apartado, desde la perspectiva históricosocial, un panorama general de ciertos recortes de subjetividad de género en el pasado. Se tomarán como fuentes de información lecturas propias y de la producción del equipo de investigación, sumado a los interesantes e insoslayables trabajos de las 
historiadoras argentinas, arraigados hace tiempo en nuestro medio y que han contribuido a esclarecer, con sus investigaciones, el pensamiento actual sobre los estudios del género y de la historia de las mujeres. Este panorama nos permitirá formular los cambios que va sufriendo el discurso en el tiempo en el tiempo (Narvaja de Arnoux, 2006, p. 28).

El trabajo de relevamiento, para el análisis que sigue, es consecuencia de la revisión de trabajos precedentes, sobre todo de un grupo de historiadoras argentinas: Mirta Lobato (Lobato, 2008, 2007, 2005, 2001, 1996), Dora Barrancos (Barrancos, 2007, 2006, 2002, 1997, 1990), Marcela Nari (Nari, 2004, 1998, 1995), Susana Torrado (Torrado, 2007, 2003, 1992); Gil Lozano (2000), entre otras, quienes han inspirado algunas de nuestras hipótesis y han guiado, en parte el análisis de estos fenómenos.

En cuanto al período de estudio, nos atenemos, de modo novedoso, a un esquema periódico que contempla las transformaciones sociales consideradas relevantes en el cambio de tendencias, que a juicio de nuestra hipótesis de trabajo, obedecen claramente a los determinantes histórico-políticos, que se corresponden con la primera mitad del siglo XX. Se tomará la periodización del sociólogo argentino Germani (1964, 1955), para el periodo histórico que nos ocupa, acentuando en nuestra lectura el rol de la mujer en cada período. El objetivo es recorrer el siguiente esquema:

a) Periodo conservador (1900-1916), que describe la incorporación de las primeras mujeres al movimiento anarquista y socialista, junto con las primeras apariciones de las mujeres en los ámbitos públicos (feministas y universitarias);

b) Periodo de democracia ampliada (1916-1930), que comprende la incipiente participación política de la mujer en las luchas sociales, que muestran el desafío de un rol activo, pródigo en asociacionismo femenino;

c) Período de la crisis mundial y del modelo agroexportador argentino (1930-1955) donde la prevalencia de la familia y el modelo eugénico se articulan con el "ideal moral" de la época;

d) Período del peronismo (1946-1955), que comprende el proceso de industrialización y desarrollo de capitales nacionales, que junto al sufragio femenino y la realización profesional logrará su esplendor, a partir de los años sesenta. 


\section{Período conservador (1900-1916). La lucha por los derechos. Mujeres anarquistas, socialistas y las primeras universitarias.}

Durante las primeras décadas del siglo $\mathrm{XX}$, continúa el proceso inmigratorio iniciado en Argentina, alrededor de 1870. En el país se produce un fuerte impulso de la industria (las fábricas y los talleres), la instalación de los ferrocarriles y el desarrollo del mercado agroexportador. Los inmigrantes llegados al territorio forman parte del plantel laboral pero no tienen derechos políticos. Es un período de reivindicaciones sociales, encabezadas principalmente por los inmigrantes anarquistas y socialistas que luchan por los derechos civiles, políticos y sociales. Las mujeres comienzan a denunciar su sometimiento. Se puede recordar como antecedente la huelga de inquilinos ocurrida en 1907, donde mujeres armadas con escobas salieron a enfrentar a la policía, que venía a desalojarlos de los conventillos. Tienen pocos hábitos de higiene y suelen tener relaciones sexuales tempranas al igual que los varones. Son consideradas "plagas sociales" ya que se vaticina para ellas un futuro de delincuencia y prostitución.

El positivismo como doctrina científica dominante instala la temática femenina desde diversos ángulos de análisis. Coinciden diversas historiadoras (Barrancos, 2007, 2006; Torrado, 2003; Falcone, 2012, 2008) que la "mujer delincuente", "la prostituta" y aún la "mujer normal" serán explicadas como degeneraciones atávicas. Para la teoría lombrosiana predominante en el ámbito médico-científico, la mujer pasaría a ocupar un lugar inferior en la escala evolutiva por su debilidad e inteligencia menos desarrollada. Las niñas callejeras son consideradas como futuras delincuentes o prostitutas mientras que las obreras son vistas como "híbridos degenerados", un elemento desintegrador del hogar.

En contraposición, las fuentes consultadas señalan roles diferentes para las mujeres pertenecientes a la elite más alta de la sociedad. El Clero y las Damas de Beneficencia asisten a la pobreza, a través de Sociedad de Beneficencia, La Escuela para mujeres, el Patronato de Menores, los asilos para ancianos y las ligas dirigidas por médicoshigienistas (Barrancos, 2006, 2007; Bellucci, 1997). Las mujeres de las clases altas controlan desde una actitud de filantropía moral poniendo énfasis en la atención materno-infantil desde el asistencialismo. En 1875 , se había promulgado una ordenanza que hacía de la prostitución una actividad legal. Las prostitutas debían registrarse, someterse a controles sanitarios, y tenían limitados algunos derechos y debían ejercer en lugares autorizados. A principios de 1900, la reglamentación queda impugnada porque lejos de prevenir y controlar favorecía la expansión del mal, declarando ilegales los prostíbulos. 
La vida religiosa de las monjas regulada por el poder eclesiástico, impone el encierro con el propósito de cuidar su virginidad, evitar las tentaciones y garantizar la pureza. La monja no ocupa lugares de poder, no trabaja y tampoco cumple con el rol de madre. Pierden el contacto con su familia, no participan de conversaciones superfluas, deben permanecer en silencio y abstenerse de mirar a los ojos de un hombre. Mientras los monjes se volcaban a la sociedad, las monjas quedaban enclaustradas. Poco a poco y entrado el nuevo siglo, las religiosas se plantean el rol asistencial. Se dedican a moralizar y a evangelizar, se comprometen con la realidad social y se dedican a atender enfermos y a educar huérfanos y niños abandonados.

Al mismo tiempo, el fin del siglo XIX y comienzos del $X X$, es testigo, aunque de modo excepcional, del ingreso de las mujeres a los estudios universitarios. Sobresalen en carreras como obstetricia, odontología y farmacia. Por entonces, tres mujeres se recibieron de farmacéuticas y aspiraron a ingresar en Medicina: Cecilia Grierson (en 1883), Julieta Lanteri (recibida en 1897) y Fanny Bonchard (1898). Elida Passo se gradúa en Suiza y luego regresa a la Argentina.

En cuanto a la presencia cívica, el Código Civil argentino, sancionado en 1871, declaraba que, al casarse,

“(...) las mujeres perdían su independencia y debían ser autorizadas por sus maridos - tal como los menores de edad tutelados o los incapacitados por enfermedad mental- para celebrar contratos o desistir de contratos anteriores y para adquirir, vender o administrar pequeñas sumas destinadas al funcionamiento hogareño sin necesidad de mediación del esposo. De manera paradojal no se impedía que las mujeres mayores de 22 años, si eran solteras, o bien las viudas o divorciadas, ejercieran el control de sus bienes, sin que requirieran el control de un varón (Código Civil, art. 52-55)" (Di Liscia, 2003, p. 92)

La expresión tácita de esta diferencia era que las mujeres mayores de edad, con bienes que administrar, y las solteras, viudas 0 divorciadas, constituían una minoría para quienes no era necesario legislar, ya que la mayoría femenina pasaba del control paterno al del marido.

El derecho al voto era masculino y de una minoría ilustrada, hasta la ley Saenz Peña de 1912, y se suceden, a lo largo de este período, las prédicas que reúnen a mujeres socialistas y anarquistas, ya que los derechos cívicos concentran, en su primera ofensiva los sectores obreros de la inmigración. Agrupaciones como Unión y labor para el progreso femenino (1900), Asociación de Universitarias argentinas (1902), Centro feminista de Buenos Aires y Liga de Mujeres librepensadoras (1905), entre otras, se suman a agrupaciones 
femeninas sufragistas: Centro Socialista Femenino (1902), Comité Pro-sufragio femenino (A. Moreau de Justo), el Centro Femenino Anarquista (1907).

\section{Período de 1916 a 1930. Democracia de participación ampliada. Intentos de liberalización civil de la mujer. Ascenso social a través de la educación y la movilidad laboral.}

Hacia 1916, se producen las primeras elecciones nacionales en Argentina. A partir de la Ley Saenz Peña (1912), se declara el voto secreto y obligatorio para los varones adultos. Dicha norma se concreta en las elecciones nacionales de 1916, iniciando el primer período democrático argentino, con la primera Presidencia de Hipólito Irigoyen, hasta 1930. Las mujeres no tuvieron a partir de esta ley el derecho cívico al voto. Aumenta considerablemente la representación de los socialistas en Buenos Aires y con el sufragio, las primeras rupturas entre feministas y antifeministas. Las mujeres votan ya por entonces en EE.UU, Australia, Nueva Zelanda, Finlandia, Alemania y Rusia.

En Argentina, el sufragio femenino contiene varios problemas de los cuales el más importante trae aparejado la liberación de las mujeres, pues a través del voto obtendrían igual ciudadanía que los varones. A partir de este planteo, la maternidad se convierte en el elemento problemático, crea el ámbito natural e iguala a las mujeres de las distintas clases sociales. No obstante, los argumentos no dejan de aparecer: la maternidad no implica la pérdida de derechos civiles, sociales y políticos; la política es una práctica destinada a las mujeres que pueden ejercerla en el hogar y en su función materna, en tanto son las responsables de la crianza de los trabajadores del mañana. Alicia Moreau de Justos os tiene un argumento contrario: la educación, la asistencia social, la justicia, la salud son ámbitos donde la mujer podría tener su lugar (conforme citado por Barrancos, 2007).

La incipiente participación política y las luchas sociales, durante este período, muestran el desafío de un rol activo para la mujer. La educación da lugar a la paulatina aparición de las mujeres intelectuales, escritoras, docentes y profesionales y aún las universitarias médicas, que hasta ahora eran una excepción. Las mujeres que habían trabajado hasta entonces en su casa - o fuera de ella como trabajadoras domésticas- comienzan a ocupar un rol diferente, a medida que comienza a emerger la mujer obrera, dedicada sobre todo al trabajo fabril. Estos cambios traen consigo un nuevo papel para las mujeres de la oligarquía porteña que, desde las Organizaciones de Beneficencia, comienzan a asistir a las obreras en la lucha por sus derechos. 
En 1924, se modifica la legislación del trabajo de mujeres que data de 1907. Las trabajadoras podrían descansar un mes antes y un mes después del parto. Se obligaba a los patrones a instalar cunas en el lugar de trabajo. En 1926, después de varios proyectos frustrados, se sanciona la ley 11.357 de derechos civiles femeninos (reformulando el Código Civil de 1871, en sus art. 52-55), por la cual las mujeres solteras, viudas o divorciadas mayores de edad pasan a ser jurídicamente iguales a los varones. Las madres casadas podrían mantener la patria potestad sobre los hijos de un matrimonio anterior y también las madres solteras. Tanto la legislación laboral específica para mujeres como la sanción de derechos civiles se amparan en el principio del bienestar de los hijos. Así se considera que una mayor independencia económica de las madres, así como un mayor poder legal sobre los hijos, redundaría en beneficio de la infancia puesto que los padres no siempre habían demostrado cumplir con sus deberes de manutención y educación.

Son varias las iniciativas por la lucha de los derechos cívicos, en este período, como el primer proyecto presentado al Parlamento Nacional (1919), o el simulacro de voto femenino realizado en Santa Fe (Argentina, 1921),o el grupo de mujeres que funda el Centro de Estudios Sociales Argentino, encabezadas por Juana Rouco Buela, o el reconocimiento de derechos cívicos iguales al hombre en la Provincia de San Juan (Argentina, 1928), que quedarán anulados a partir del inicio del conservadurismo de Uriburu, después del golpe de estado de 1930, que silencia casi por toda la década las posibilidades de reclamos.

\section{Crisis mundial y del modelo agro-exportador argentino (1930- 1946). La mujer y la familia en su rol de protección de la raza. Modelo eugénico.}

Hacia la década del '30, el panorama político, económico y social había cambiado. El contexto internacional estaba cruzado por el afianzamiento del Stalinismo en la Unión Soviética, la emergencia y consolidación del nazismo en Europa, el comienzo de la Guerra Civil española y el inicio de la Segunda Guerra Mundial.

En Argentina, se restablece el conservadurismo con el apoyo de los militares. Se produce el primer golpe de Estado junto con el agotamiento de la economía agroexportadora. Los cambios políticos y económicos acompañan las transformaciones poblacionales. Las migraciones internas inician un lento proceso de vaciamiento de las provincias argentinas. La inmigración que se había sostenido, desde el inicio de su proceso hacia 1880, empieza a declinar durante el Gobierno de facto de Uriburu. Se endurecen las trabas al ingreso de 
inmigrantes. La crisis económica nacional fue tan vasta que al período se lo conoce como "década infame" (Romero, 2006).

En el marco de una profunda crisis económica y social, el golpe nacionalista del '30 abre un período de democracia restringida. La eficacia de los gobiernos nacionalistas debía quedar demostrada por su capacidad para enfrentar la difícil situación económica. El conjunto de la economía fue cerrándose progresivamente con una intervención cada vez mayor del Estado. El cierre de la economía y los efectos de la Segunda Guerra Mundial favorecieron el desarrollo industrial especialmente en el sector alimentario y textil (Germani, 1964).

$\mathrm{Si}$ bien en estas décadas hubo un aumento considerable de las obreras, la incorporación de la mujer al mundo del trabajo siguió siendo conflictiva pues generaba tensiones con las obligaciones familiares. Los discursos continuaron enfatizando el lugar de realización de la mujer en la maternidad. La obrera seguía siendo un elemento disgregador de la unión del hogar.

La intervención social del Estado se concreta en dos áreas ligadas a la mujer: la delincuencia y la prostitución. Se crea por decreto el Patronato Nacional de Menores que, junto con las instituciones de caridad (Damas de la Sociedad), condujo a la formación de hogares para niñas. Hasta 1930, la formación de las niñas de la calle estaba a cargo de monjas que las preparaban en las tareas domésticas. A partir de 1932, se crea una institución del Patronato que preparará a las niñas para conseguir trabajo como obreras o empleadas. La época de encarcelar a las menores había pasado, se empieza a admitir el comercio y la industria como lugar de regeneración de las jóvenes marginales (Torrado, 2003). Hacia fines de 1936, se sanciona la Ley 12.331, conocida como Ley de Profilaxis de las enfermedades venéreas. Si bien la meta manifiesta era prevenir y tratar enfermedades sexuales, la misma tenía otra intención prohibir la prostitución. De esta manera la ley en cuestión daba por terminado un período iniciado a fines del siglo XIX, en que se había impuesto un criterio reglamentarista de dicha actividad.

En este período la idea de la familia como "célula básica" de la Nación permanecía inmutable. Por ello cualquier trastorno que impidiera el sano desenvolvimiento de las familias debía de ser atacado. Si bien la idea de la mujer como única agente de contagio se había visto superada por diversos informes científicos, aún se sostenía.

A principios de los '40, diversas fuentes señalan con preocupación el retraso en el ingreso al matrimonio. ¿Como sostener un noviazgo decente sin dar lugar a actos deshonestos? Noviazgos largos, trabajo femenino y Ley de Profilaxis daban lugar al amor libre o clandestino. En otras palabras, se había creado una situación potencialmente inmoral, propiciada por la ley, donde las mujeres decentes mancharían su reputación. Entre el año de la sanción de la Ley y el inicio de la década del '40, se evidenció un retroceso de la sífilis, pero 
a partir de 1940, desde distintos seminarios médicos se alerta sobre el pavoroso incremento de la enfermedad (Barrancos, 2006, 2007; Torrado, 2003; ).

En el campo médico, la asistencia y protección maternal debió encarar las más complejas cuestiones biológicas, técnicas y sociales de aquella ciencia: afianzamiento de los principios de indestructibilidad del binomio madre- hijo, la actividad preventiva de las enfermedades de la futura madre, la lucha contra las enfermedades sociales y del trabajo y simultáneamente: la investigación de las causas del infanticidio, la represión del aborto, el reconocimiento médico pre matrimonial, el consejo prenupcial, el certificado prenupcial, el permiso matrimonial y la carta de familia.

Las mujeres acompañan la crisis saliendo a trabajar, emergiendo las obreras y empleadas como las nuevas protagonistas. Simultáneamente las estadísticas comienzan a mostrar un paulatino y continuo descenso de la natalidad. En este estado de cosas las normas referidas a la mujer enfatizan su inclusión en el mercado laboral. Obtienen derechos civiles y en el campo productivo gozan de una política de protección para la mujer embarazada, con licencia 30 días antes y después del parto (Novick, 1993). En lo sanitario se protege a las mujeres del contagio y las enfermedades exigiendo el certificado prenupcial masculino. Se cierran los prostíbulos en toda la República y se dictan normas que protegen el matrimonio. El Estado declara expresamente que garantiza la salud del binomio madre-hijo y elabora una ambiciosa política sobre el tema. Novick (1993) sostiene que si bien estas normas persiguen en primera instancia el combate de los males venéreos, lo hacen bajo el ideal de fomentar los matrimonios, combatir la de natalidad y propender a la salud de la raza.

Emparentado con la política del Estado, los médicos adhieren a los modelos eugenésicos. Dora Barrancos (Barrancos, 2007) sostiene que el peso del eugenismo asumió diversas modalidades - aspectos alimentarios, programas de vida sana, derecho a limitar el número de hijos- mostrando una clara preocupación por la descendencia y los problemas de población, en el marco de un agotamiento del modelo agroexportador. El pensamiento eugénico desempeñó una clara influencia en el discurso de género en torno a la maternidad. La maternidad biológica se situó en relación directa con la preocupación de la degeneración de la raza y la despoblación. Las mujeres garantizaban la procreación y sobrevivencia de las futuras generaciones ${ }^{4}$. 


\section{El peronismo (1946-1955). El sufragio femenino, participación política y realización profesional.}

Luego de un largo período en que se sucedieron gobiernos militares y represión policial, se inaugura, en 1946, un período de democracia de participación ampliada (Germani, 1964), hasta el golpe de estado de 1955. A través de la restitución del voto secreto y obligatorio para varones adultos se elige, por dos mandatos, a Juan D. Perón como Presidente de la República Argentina. Con la llegada de Perón surge la figura de Eva quien se convertiría en una figura política central para los sectores postergados o discriminados, la ancianidad, la niñez y las mujeres.

En 1949, Evitase convierte en la Presidenta del Partido Peronista Femenino (P.F.P), como partido autónomo exclusivamente integrado por mujeres. Desde el partido se selecciona un grupo de 23 mujeres, que se convertirían en delegadas censistas para organizar unidades básicas, que consistían en agrupaciones fundamentalmente dirigidas por mujeres, con el propósito de brindar asistencia social a los pobres, a los niños y a los jubilados. Eran amas de casa, maestras o enfermeras. Todas jóvenes y admiradoras de la figura de la líder peronista (Dos Santos, 1983). El partido es considerado una gran familia y cada una de sus integrantes son mujeres al servicio de la Nación (Toppi, 2006).

En 1946, Eva Perón había presidido la Comisión Pro-sufragio femenino y empieza a presionar para que la ley se sancione. El 9 de setiembre de 1947, el Congreso de la Nación Argentina promulga la Ley $\mathrm{N}^{\circ}$ 13.010, que otorga los derechos políticos a las mujeres. La firma del Decreto Ley es un gesto simbólico, convirtiéndose la líder peronista en la abanderada de las mujeres. El 11 de setiembre de 1951, la mujer argentina emite su voto por primera vez significando un hito cívico en la ciudadanía argentina (Valobra, 2009). Otras mujeres habían sentado las bases para dicha Ley: Cecilia Grierson, Petrona Eyle, Julieta Lanteri, Alicia Moreau, Sara Justo Raquel Camaña y Elvira Rawson, quienes habían cuestionado ya la condición civil, la exclusión ciudadana y la falta de educación. En 1910, se había llevado a cabo el Primer Congreso Femenino Internacional. Desde 1911 hasta 1947, se presentaron 15 proyectos de Ley, que fueron todos archivados por la mayoría conservadora y también por las divisiones ideológicas de las feministas argentinas que no lograron efectivizar una acción conjunta.

Bajo la vigencia del modelo peronista las trabajadoras obtienen mejoras económicas, protección social y vacaciones. Pese a estos logros, afirma Barrancos (2007) el ideal maternal sigue siendo la meta primordial de las mujeres.

Las reformas en lo que concierne al campo de la salud bajo el modelo peronista resultan trascendentes, más aún si pensamos su 
articulación con el desarrollo de nuestra propia disciplina que asiste a su creación en esta década. El 23 de mayo de 1946, se crea la Secretaría de Salud Pública, que en 1949, se convierte en Ministerio. El Dr. Ramón Carrillo es designado como el primer Secretario de Salud Pública con rango de Ministro de Salud Pública de la Nación. Al hacerse cargo del Ministerio el panorama era una gran desorganización, desmantelamiento de las instalaciones sanitarias, falta de camas, de médicos y personal capacitado. La política sanitaria implementada se basó en tres áreas completamente nuevas: Medicina Preventiva, Medicina Social y Atención materno infantil. El Plan Quinquenal (1947-1951), en su apartado sobre la Salud Pública, apunta a la salud gratuita y erradicación de las enfermedades regionales, asistencia a la maternidad, a la infancia y a la vejez. También estimula la investigación en medicina higiénica y preventiva, la profilaxis y los tratamientos. El plan asistencial fue muy ambicioso pero también muy exitoso. Se basó en tres puntos fundamentales: funcionamiento hospitalario, construcción de hospitales y división del país en zonas sanitarias. Con estos tres puntos daría prioridad a la medicina preventiva y a la modernización hospitalaria. Ligado a la gestión de Ramon Carrillo se han publicado numerosos artículos que consideran que la acción del Ministerio de Salud Pública generó las condiciones propicias para la creación de carreras humanísticas en los 50: Psicología, Antropología, Ciencias de la Educación y Sociología ${ }^{5}$.

\section{DISCUSIÓN}

Considerando la incidencia de algunas de las variables políticas, sociales y económicas el presente artículo se propuso mostrar un panorama de las diversas teorías interpretativas, que describen las modalidades de inserción social y participación social de la mujer, entre fines de siglo XIX y mediados del XX. Aún cuando, por razones de espacio, no pudieron incluirse la multiplicidad de factores intervinientes, el esquema de periodización trabajado recoge la diversidad de formulaciones discursivas y representan los cambios de tendencia buscados.

El período analizado, aun cuando su abordaje no haya sido exhaustivo, está justificado en que la primera mitad del siglo XX, fue una época de importantes cambios en la sociedad argentina, que contribuyeron profundamente a la construcción de la subjetivación femenina. El análisis de las ocupaciones, la política y la participación, la familia, las fábricas, los conventos, las clases sociales han podido revelar los modos de presencia de las mujeres en la esfera pública, tanto desde su significativa diversidad como por su articulación a la época. 
La discusión que surge luego del análisis de las fuentes consultadas y trabajos propios precedentes- afianzan la formula, no por ligera menos esclarecedora, que plantea un principio dinámico, desde el abandono paulatino de los roles tradicionales de la "mujer destinada al hogar" a los de la "mujer sostén del hogar", aplicable a las sociedades contemporáneas.

Hacia principios de siglo XX, la mujer afectivamente dependiente, frágil y emotiva construye su rol alrededor del hogar y la maternidad diferenciándose de las mujeres "fuera del hogar" relegadas a la prostitución, el contagio y la enfermedad. Los primeros desempeños en ámbitos públicos implicaron a las mujeres en tareas de atención a la pobreza y la infancia. Las Damas Patricias, la Escuela de mujeres, el Patronato de menores, la asistencia pública, etc. son instituciones que albergan mujeres para tareas altruistas o filantrópicas, que no van más allá, según nuestras reflexiones, de una prolongación de los roles hogareños tradicionales (cuidar, amparar, enseñar), y que obedecen a un sistema de intervención directa (sistema educativo) o indirecta (filantropía) del Estado Conservador. De hecho una de las primeras instituciones creadas fue Unión y labor para el progreso femenino y protección del niño (1900).

En la misma época, aunque de manera excepcional, las mujeres se incorporan a los estudios universitarios sobresaliendo en carreras como medicina, obstetricia, odontología. Aún cuando estas acciones pertenecen indudablemente a la esfera pública no se apartan del destino de género de las sociedades patriarcales. Por otro lado, varios de los autores consultados plantean la discusión alrededor del papel que tuvieron las mujeres en la educación y el cuidado de la salud de la población, acentuando la acción de maestras y enfermeras, que confirman la idea de que la mujer, por su mayor "sensibilidad" o emotividad, tiene la misión de cuidar y consolar a los que la rodean (Morgade, 1997; Wainerman, 1980; Falcone, 2012).

Aun con estas primeras reflexiones un recorrido por la primera mitad del siglo XX, encuentra a las mujeres envueltas en discusiones sobre el sufragio femenino, los derechos ciudadanos y las primeras luchas por las reivindicaciones laborales. En efecto, los sucesivos cambios económicos obligaron a las mujeres a salir del ámbito hogareño e involucrarse en la esfera pública para formar parte de la lucha en organizaciones gremiales, huelgas y manifestaciones, denunciando la precaria situación laboral y de desigualdad respecto de los hombres. Hemos destacado los debates que se plantean, en varios de los estudios de referencia, sobre aquellas mujeres que se opusieron a los mandatos patriarcales de su época y aquellas que ocuparon puestos masculinos; la acción de los primeros movimientos feministas, la participación de las mujeres en las prácticas de ideologías como el anarquismo, el socialismo y el peronismo, el asociacionismo femenino, y el trabajo femenino (Lobato 2001, 2000; Barrancos, 
2007, 2002, 1997, 1990; Nari, 2004, 1995; Torrado, 2003, 1992; Sautu, 1980), estableciendo sus diferencias.

De los relevamientos realizados surge la pregunta sobre los cambios de tendencia coincidentes con los gobiernos conservadores. Nos referimos al estudio comparativo que puede hacerse entre el primer período (1900-1916) y el tercer período (1930-1946), este último en que se suceden gobiernos militares. Se advierte el énfasis puesto en la idea de familia, en la realización de la mujer en la maternidad y en el hogar como soporte de una nación sana. En ambos períodos de democracia restringida, la concurrencia de los discursos religioso, político, médico-psiquiátrico y legal dan como resultado el fuerte retroceso de las mujeres del ámbito público.

Asimismo, nos preguntamos, luego de nuestro propio relevamiento, si el trabajo y la educación no se constituyen en dos claves para entender los cambios de tendencia, que marcan la dinámica de la inclusión femenina "en el tiempo". Siguiendo, en parte, a nuestros autores de referencia se podría plantear la discusión en los siguientes términos: en tanto que las nuevas formas de organización económica y los procesos de industrialización y modernización del siglo XX, plantean la paulatina separación entre la familia y el trabajo, entre la producción doméstica y las formas socializadas de producción, esto no trae aparejado la redefinición de las relaciones familiares y la división sexual laboral. En efecto, desde el momento en que la familia, deja de ser una unidad de producción para transformarse en una de tipo emocional, la obtención material de bienes pasa a realizarse fuera del hogar y se reformulan las anteriores relaciones entre hombres y mujeres. Los componentes básicos de esta ideología son analizados por varios autores en términos de disociación entre el "mundo de lo público y de lo privado" (Lobato, 2008; Guy, 1998.). Varias son las autoras que despliegan estos debates (Gil Lozano, Pita, \& Ini, 2000; Nari, 1998; Torrado, 2003; Novick, 1993; Lobato, 2007) y coinciden en afirmar que son estos cambios ligados a los procesos económicos, los que comienzan a vincular a la mujer con el ámbito doméstico, con la ausencia de decisión fuera de la familia y los hijos, y al hombre con el ámbito público, con la toma de decisiones, con el poder económico y político. La misma visión es compartida por algunas feministas contemporáneas cuando identifican el espacio público con la productividad económica y al espacio privado o doméstico con la baja o nula remuneración.

Ahora bien, las primeras actividades laborales femeninas, si bien se sitúan en la esfera pública, repiten los roles esperables: lavanderas, cocineras, trabajadoras domésticas, nodrizas, costureras y maestras dentro del hogar o fuera de él (Zurita, 1981; Wainerman \& Recchini, 1981; Wainerman \& Navarro, 1979; Lobato, 2008). A medida que el país ingresa en la etapa industrial, el crecimiento vertiginoso de la población y la demanda de bienes producen el ingreso de las mujeres 
como obreras en las fábricas textiles, de cigarrillos, mataderos; o empleadas en el comercio y la telefonía (Feijo, 1990). Por lo que serán estos nuevos roles los que favorecen las primeras luchas por los derechos y las conquistas sobre las reglamentaciones del trabajo femenino.

En cuanto a la educación, hay varias investigaciones (Bellucci, 1997, 1990; Morgade, 1997) que vamos a discutir brevemente. Dichas indagaciones coinciden en afirmar, que solo las mujeres que accedieron a una mayor escolaridad pudieron incorporarse a la docencia como alternativa al trabajo doméstico y al industrial. En el mismo sentido, Wainerman y Lavrin (Wainerman, 1980; Lavrin, 2005) señalan una tendencia marcada por el ingreso de mujeres de clase media, y con mayores niveles de educación a actividades como la administración, los servicios sociales, las finanzas y los seguros, a medida que se produce un crecimiento paulatino del sector terciario (salud, educación, gobierno). Asimismo, las mujeres con menor escolaridad logran acceder al sector comercial, administrativo y de servicios, como alternativa al trabajo doméstico o rural. No obstante, Nari (1998) plantea, al respecto que las tareas de docencia, administrativo, trabajo doméstico y de servicio (en contraste con la actividad industrial o rural) son actividades consideradas "feminizadas", en tanto quedan asociadas a las tareas hogareñas.

La última etapa (1946-1955) nos plantea una nueva discusión sobre los cambios de tendencia, en los modos de inclusión de las mujeres en la esfera pública, considerada hasta entonces eminentemente masculina. En los años 50 y con mayor énfasis en los 60, la discusión es nueva, estrechamente vinculado al despegue económico y a ciertos determinantes sociológicos (movimientos de liberalización femenina etc., nacionales e internacionales) se abre una época de cambios, que marcan un hito indiscutible, en relación con la independencia femenina y la inclusión de los hombres en las tareas del hogar. Por esta época, dice Barrancos (1991), las mujeres sufragistas, reformistas, asistencialistas (primeras décadas de nuestra periodización) abogan juntas contra la contracepcionalidad y a la posibilidad de desarrollar su sexualidad libremente. Del mismo modo, el cambio de tendencia se expresa en el trabajo, puesto que la inserción laboral pasa a ser un factor de independencia económica y de realización personal y no simplemente un paliativo para ayudar a resolver la economía familiar. Las mujeres empleadas o profesionales fueron las primeras que vieron en el trabajo la emancipación económica además de un ambiente propicio a las relaciones sociales alternativo al hogar. 


\section{CONCLUSI ONES PRELI MI NARES}

Las primeras conclusiones a las que se ha podido arribar afianzan la idea de que las transformaciones sociales producidas, desde principios de siglo XX hasta la década del 50, han contribuido a la construcción de la subjetividad femenina, que ha ido cambiando conforme las vicisitudes económicas y políticas. Los distintos momentos de periodización trabajada reflejan cambios de tendencia en el estudio de los roles de la mujer y sus variaciones en el tiempo. Dichos cambios marcan un principio de evolución que representa el abandono paulatino de los roles hogareños de las sociedades patriarcales hacia la conquista de la esfera de lo público (no doméstico). En este pasado, aunque no sin dificultades, arraigan los movimientos de lucha por la ampliación de derechos que podemos ver representada en las discusiones actuales, de las sociedades contemporáneas.

La periodización analizada refleja los cambios de tendencia en el estudio de la "mujer en el tiempo", coincidentes con las transformaciones políticas, en periodos de democracia ampliada (1916-1930/1946-1955), o bajo el régimen de los gobiernos conservadores (1900-1916/1930-1946). La lucha por los derechos y la incipiente participación política muestran el desafío de un rol activo, pródigo en asociacionismo femenino, que se expresa en plenitud, en los períodos de democracia ampliada. Mientras que en los períodos de gobiernos conservadores el retroceso en esas conquistas de derechos se hace evidente.

Las prédicas sobre la dependencia masculina (padre o esposo), o el derecho al voto masculino (Ley S. Peña/1912), o de una minoría ilustrada, reúne a mujeres socialistas y anarquistas, en defensa del sufragio femenino, especialmente en la década del 20 . El seguimiento de la aparición de las primeras agrupaciones femeninas sufragistas, desde 1902, hasta el simulacro de voto femenino realizado en Santa Fe (Argentina), en 1921, o el reconocimiento de derechos iguales al hombre en la Provincia de San Juan (Argentina) comprueba que, a partir del golpe nacionalista de 1930, y el conservadurismo de Uriburu, se ven anuladas las posibilidades de reclamo, las mujeres son eliminadas del padrón electoral y las leyes mantienen a nuestras mujeres en el hogar y al cuidado de los hijos. Serán las mujeres "peronistas", en el segundo período democrático (1946-1955), de un origen diferente a las primeras feministas, las que lograrán en 1947, el reconocimiento de la ciudadanía (Ley 13.010), promocionada por Eva Perón y votada por el Congreso Nacional.

El mismo análisis puede aplicarse al mundo laboral de la mujer, que repite los roles establecidos en su destino de género: maestras, nodrizas, trabajadoras domésticas, etc. Si bien la presencia de la mujer es aceptada en el mundo del trabajo, su inserción en este 
universo queda asociada a las dificultades y obstáculos que trazan un movimiento ambiguo y zigzagueante. En todos los casos, el trabajo no es considerado una realización personal de la mujer en su profesión u ocupación, sino una respuesta a una necesidad económica apremiante, planteada por las demandas de la estructura económica en cambio. Solo el último período democrático de nuestro análisis marca un hito en la plena ocupación femenina en la esfera laboral y en la adquisición de derechos laborales junto a la inclusión plena en carreras terciarias o profesionales.

El siguiente grupo de conclusiones reflexiona sobre las contradicciones entre el rol social y público de la mujer y su rol familiar, coincidente con los gobiernos conservadores de la década del 30. Las mujeres retroceden de la esfera pública, lo cual tiene su explicación en la prevalencia de los criterios naturalistas y biotipológicos de la medicina eugénica, que logra imponer, aunque veladamente, el ideal moral en la sociedad. Pudimos ver en este periodo las enormes proyecciones de la maternidad, en relación con la eugenesia, y como la iglesia colabora en ese proceso asignando a la mujer - futura madre- la función de transmitir los valores católicos, que al mismo tiempo son funcionales al modelo capitalista.

La medicina eugénica instruye comportamientos a través de los manuales de higiene, pedagogía doméstica, puericultura y urbanidad que circulaban entre las mujeres de la época. Las madres en representación de las familias y los médicos, representantes sanitarios del Estado, controlan las problemáticas sociales bajo un sistema de alianzas que fortalece el ideal de familia. Virtudes burguesas como el trabajo, la honradez, responsabilidad, buenos modales debían ser transmitidos por las mujeres en el hogar.

En nuestro análisis, los años '30, han mostrado su costado ambivalente y contradictorio. La discusión se plantea entre el conservadurismo de la sociedad que propugna el lugar tradicional de la mujer - ama de casa y reproductora genética- y al mismo tiempo, se observa la continua incorporación de las mujeres al mercado laboral y se patentiza en la declinante fecundidad y despoblación. Las paradojas de la década, por un lado, elevan el rol de la mujer en su función de madre, y por otro lado, contradictoriamente las medidas de reconocimiento y fomento del trabajo femenino son innumerables. Por último, en la etapa peronista, con las elecciones nacionales en 1946, los derechos políticos adquiridos, junto a la sindicalización de las mujeres, encuentra a las mujeres en un rol completamente nuevo. Estas mujeres, identificadas con Evita, de un origen más humilde que las primeras feministas, protagonizan el trabajo social, se comprometen con las leyes de protección a la mujer y a los niños, y aportan modelos de educación, salud y previsión. La inclusión femenina en esta etapa es de plena participación en el mercado laboral y en la oferta profesional y universitaria. No obstante, en un 
análisis en el tiempo, se destaca que el reconocimiento de las desigualdades laborales y sociales son conquistas anteriores a los derechos civiles, puesto que como se pudo ver el sufragio femenino, en nuestro país, se produce tardíamente - las mujeres votan por primera vez, en 1951.

Finalmente, destacamos la masiva incorporación femenina a las carreras terciarias y universitarias, en el transcurso de la década del 50 y con más fuerza en los 60 , que justamente se corresponde con la época en que se crean, en Argentina, las nuevas carreras de Psicología, tanto en la Universidad de Buenos Aires, como en la Universidad de La Plata y en el interior del país, albergando a una mayoría abrumadora de mujeres entre sus alumnas y profesoras. Época que a todas luces significó el reconocimiento profesional de nuestras mujeres que hasta hoy pueblan en mayoría las aulas de nuestras universidades.

\section{Referencias}

Barrancos, D. (2007). Mujeres en la sociedad argentina. Una historia de cinco siglos. Buenos Aires: Sudamericana.

Barrancos, D. (2006). Las mujeres y sus luchas en la historia argentina. Buenos Aires: Paper Ministerio de Defensa. Recuperado de https://es.scribd.com/document/131153894

Barrancos, D. (2002). Inclusión/ exclusión: Historia con mujeres. Buenos Aires: Fondo de Cultura Económica.

Barrancos, D. (1997). Presencia de la mujer en las luchas sociales argentinas de principios de siglo. Aportes para una Argentina plural. Archivo General de la Nación, 1(2), 117-128.

Barrancos, D. (1991). Contracepcionalidad y aborto en la década del 20: problema privado y cuestión pública. Estudios Sociales, 1(2). Recuperado de http://www. academia.edu/4682936

Barrancos, D. (1990). Anarquismo, educación y costumbres en la Argentina a principios de siglo. Buenos Aires: Contrapunto.

Bellucci, M. (1990). Anarquismo, sexualidad y emancipación femenina. Nueva sociedad, (109), 148-157. Recuperado de http://www. academia.edu/4682936

Bellucci, M. (1997). Mujeres en la educación (1870-1930). Buenos Aires, Argentina: Miño y Dávila editores.

Bordieu, P. (1983). Campo de poder, campo intelectual. Buenos Aires: Folios.

Campbell, D., \& Stanley, J. (1970). Diseños experimentales y cuasi experimentales en la investigación social. Buenos Aires: Amorrortu.

Castoriadis, L. (1986). La constitución imaginaria de la sociedad. Buenos Aires: Tusket. 
Cook, T., \& Reichardt, C. (1986). Métodos cualitativos y cuantitativos en investigación evaluativa. Madrid: Morata.

Di Liscia, M. S. (2003). Mujeres, Locura e incapacidad civil en Argentina, 1890-1920. La Aljaba: Revista de Estudios de la Mujer, (8), 89-105.

Dos Santos, E. (1983). Las mujeres peronistas. Buenos Aires: Centro Editor de América Latina.

Falcone, R. (2016, septiembre/octubre). "Procesos de construcción de la subjetividad femenina: la publicidad como vehículo de interpretación". Actas del XVII Encuentro Argentino de Historia de la Psicología, la Psiquiatría y el Psicoanálisis (pp. 301-307). Buenos Aires, Argentina, 17. Recuperado de https: // histopsi2018. weebly.com

Falcone, R. (2015, noviembre). Estudios del género y construcción de la subjetividad. Aportes desde la historia de la Psicología en Argentina. Memorias del VII Congreso Internacional de Investigación y práctica profesional en psicología, XXII Jornadas de Investigación, XI Encuentro de Investigadores en Psicología del Mercosur (pp. 12-17). Facultad de Psicología, Universidad de Buenos Aires, Buenos Aires, Argentina. Recuperado de https: //www. aacademica.org/000-015/138.pdf

Falcone, R. (2012). Género, familia y autoridad. Sociedades patriarcales y comunidades contemporáneas. Revista Científica de UCES, 16(1), 67-73. Recuperado de http://dspace.uces.edu.ar: 8180/xmlui/bitstream/handle/12345 $6789 / 1465 /$ Genero_familia_Falcone.pdf?sequence $=1$

Falcone, R. (2011). Género, subjetividad e historia: el rol de la mujer argentina en la primera mitad del siglo XX. Revista Temas de Historia de la psiquiatría argentina, 25(25), 17-23.

Feijo, M. del C. (1990). Las trabajadoras porteñas a comienzo del siglo. In D. Armus (Comp.), Mundo Urbano y Cultura Popular. Estudios de Historia Social Argentina (pp.281-311). Buenos Aires: Sudamericana.

Germani, G. (1955). Estructura social de la Argentina. Buenos Aires, Argentina: Paidós.

Germani, G. (1964). Política y sociedad en una época de transición. Buenos Aires: Paidós.

Gamba, S. B. (2008). Perspectiva de Género. ¿Qué es la perspectiva de género y los estudios de género? In S. B Gamba (Coord.), Diccionario de estudios de Género y Feminismos. Buenos Aires, Argentina: Biblos.

Gil Lozano, F., Pita, S., \& Ini, M. G. (Comp.). (2000). Historia de las mujeres en Argentina (Vol. 2, siglo XX). Buenos Aires, Argentina: Edit. Taurus.

Guy, D. (1998). Madres vivas y muertas. Los múltiples conceptos de maternidad en Buenos Aires. In D. Balderston, \& D. Guy 
(Comp.), Sexo y sexualidad en América Latina (pp. 231-256). Buenos Aires: Paidós.

Hacking, I. (2001). ¿La construcción social de qué?. Barcelona: Paidós.

Jodelet, D. (1986). La representación social: fenómenos, conceptos y teoría. In S. Moscovici, Psicología Social II: pensamiento y vida social: Psicología social y problemas sociales (pp. 470-494). Barcelona: Paidós.

Jodelet, D. (2007). Representaciones sociales: contribución a un saber sociocultural sin fronteras. In D. Jodelet, \& L. Guerrero, Develando la cultura. Estudio en representaciones sociales ( $p p$. 7-30). México: UNAM.

Lobato, M. Z. (1984). El fenómeno de las representaciones sociales. In R. M. Farry \& S. Moscovici (Eds.), Social Representation. Cambridge: Cambridge University Press. Paris: Ediciones de la Maison des Sciences de l' home.

Lobato, M. Z. (Comp.). (1996). Política, médicos y enfermedades. Lecturas de historia de la salud en la Argentina. Buenos Aires: Biblos.

Lobato, M. Z. (2000). Entre la protección y la exclusión. Discurso maternal y protección de la mujer obrera. Argentina, 18901934. In J. Suriano (Comp), La cuestión social en la Argentina. Buenos Aires: La Colmena.

Lobato, M. Z. (2001). La vida en las fábricas. Trabajo, protesta y política en una comunidad obrera (1904-1970). Buenos Aires: Prometeo Libros.

Lobato, M. Z. (2005). Cuando las mujeres reinaban. Belleza, virtud y poder en la Argentina del siglo XX. Buenos Aires: Biblos.

Lobato, M. Z. (2007). Historia de las trabajadoras en la Argentina (1869-1960). Buenos Aires: Edhasa.

Lobato, M. Z. (2008). Trabajo, cultura y poder: dilemas historiográficos y estudios de género en Argentina. Estudios de Filosofía práctica e Historia de las ideas, 9(10), 29-45. Recuperado de http://qellqasqa.com.ar/ojs/index.php/estudios

Lavrin, A. (2005). Mujeres, feminismo y cambio social en Argentina, Chile y Uruguay. (1890-1940). Santiago de Chile: Centro Universitario Diego Barrios.

Moscovici, S. (2000). Representaciones sociales: exploraciones en psicología social. Francia: University Presses of France. doi: $10.5565 / \mathrm{rev} /$ athenead/v1n2.55

Morgade, G. (1997). Mujeres en la educación. Género y docencia en Argentina 1870-1930. Buenos Aires: Miño Dávila.

Narvaja de Arnoux, E. (2006). Análisis del discurso: modos de abordar materiales de archivo. Buenos Aires: Santiago Argos Editor. 
Nari, M. (1995). Feminismo y diferencia sexual. Análisis de la encuesta feminista argentina de 1919. Boletín del Instituto de Historia Argentina y Americana Dr. Emilio Ravignani, 3(12), 6186.

Nari, M. (1998). De la maldición al derecho. Nota sobre las mujeres en el mercado de trabajo, Buenos Aires 1890-1940. In H. B. Garrido, \& M. C. Bravo (Eds.), Temas de mujeres: perspectivas de género (pp. 97-110). Tucumán, Argentina: Universidad Nacional de Tucumán.

Nari, M. (2004). Políticas de maternidad y maternalismo político: Buenos Aires 1890-1940. Buenos Aires: Biblos.

Novick, S. (1993). Mujer, Estado y políticas sociales. Buenos Aires: CEAL.

Pastor Ramos, G. (1994). Conducta interpersonal: Ensayo de Psicología Social sistemática. Salamanca: Universidad Pontificia de Salamanca.

Romero, J. L. (2006). Breve historia de la Argentina. Madrid: Fondo de Cultura Económica.

Sautu, R. (1980). El mercado de trabajo: la mano de obra femenina, Primera Historia Integral Argentina, 55. Buenos Aires: Centro Editor de América Latina.

Scott, J. W (1990). El género: una categoría útil para el análisis histórico. In J. Amelangy, \& M. Nash, (Comp.), Historia y género: las mujeres en la Europamoderna y contemporánea. Barcelona: Alfons el Magnanim.

Scott, J. W. (1996). Historia de las mujeres. In P. Burke (Ed.), Formas de hacer historia. Madrid: Alianza Universidad.

Toppi, H. P. (2016). Políticas públicas y derechos políticos: Del voto femenino a las cuotas de género como respuestas a los problemas de representación política de las mujeres en la Argentina. Revista Perspectivas de Políticas Públicas, 5(10), 87120. doi: 10.18294/rppp.2016. 1017

Torrado, S. (1992). Estructura Social de la Argentina 1945-1983. Buenos Aires: Ediciones de La Flor.

Torrado, S. (2003). Historia de la familia en la argentina moderna (1870-2000). Buenos Aires: Ediciones de la Flor.

Torrado, S. (Comp.). (2007). Población y bienestar en Argentina. Buenos Aires: Edhasa.

Valobra, María (2009). “...Del hogar a las urnas..." Consideraciones sobre la ciudadanía política femenina, 1946-1947. e-l@tina, 7(27), 45-65. Recuperado de http://www.memoria.fahce.unlp.edu.ar/art_revistas/pr.7375/pr .7375.pdf

Van Dijk, T. (1994). Discurso, Poder y Cognición social: Conferencias de Teun A. van Dijk. Cuadernos 2(2), 1-93. Recuperado de 
http: //www. discursos.org/oldarticles/Discurso, \% 20poder\% 20y $\% 20$ cognici\% F3n\% 20social. pdf

Wainerman, C. H., \& Navarro, M. (1979). El trabajo de la mujer en la Argentina: un análisis preliminar de las ideas dominantes en las primeras décadas del siglo XX (Serie Cuadernos del CENEP, 7). Buenos Aires: CENEP. Recuperado de http://www.cenep.org.ar/index. php/publicaciones-del-cenep

Wainerman, C. H., \& Recchini de Lattes, Z. (1981). La Medición del trabajo femenino (Serie Cuadernos del CENEP, 21). Buenos Aires: CENEP. Recuperado de http://www.cenep.org.ar/index. php/publicaciones-del-cenep

Wainerman, C. H. (1980). Educación, familia y participación económica femenina en la Argentina. Buenos Aires: CENEP. Recuperado en http://www.cenep.org.ar/index. php/publicaciones-del-cenep

Zurita, C. (1981). Servicio doméstico en Argentina entre 1947 y 1970: una estimación a partir de datos censales. Montevideo: Seminario de desarrollo rural y trabajo femenino. Recuperado de http://www. cenep.org.ar/index.php/publicaciones-del-cenep

\section{Endereço para correspondência Rosa Falcone}

Universidad de Buenos Aires - UBA

Arturo J auretche, 184 (1405), CABA, Argentina

Endereço eletrônico: rofalcone@gmail.com

Recebido em: 21/12/2018

Reformulado em: 11/02/2019

Aceito em: 28/02/2019

\section{Notas}

* Doctora en Psicología (UBA). Pos-Doctora en Estudios del Género (UCES), Profesora Historia de la Psicología Universidad de Buenos Aires (UBA) y Universidad de Ciencias Empresariales y Sociales (UCES).

${ }^{1}$ Proyecto trienal UBACyT, Programación científica 2018-2020, “Configuraciones de lo femenino: metáforas, discursos y casos de la Psicología, la Psiquiatría y el Psicoanálisis, entre fines del siglo XIX y mediados del XX", Secretaría de Ciencia y Técnica, Universidad de Buenos Aires. Aprobado. Código 20020170100013BA. Director: Rosa Falcone

2 Dagfal, A. (1997). Discursos, instituciones y prácticas presentes en la etapa previa a la profesionalización de la disciplina psicológica en la argentina (1945-1955). Cuadernos Argentinos de Historia de la Psicología, 3(1-2), 173-195.; Klappenbach, H. (2006). Periodización de la psicología en Argentina. Revista de Historia de la Psicología, 27(1), 109-164; Klappenbach, H. (1995). Antecedentes de la carrera de psicología en las universidades argentinas. Acta Psiquiátrica y Psicológica de América Latina, 3(41), 237-243.; Klappenbach, H. (1995). The process of Psychology's professionalization in Argentina. Revista de Historia de la Psicología, 16(1-2), 97-110.; Rossi, L. et al. (Colabs.). (1995). Psicología: "Secuencias Instituyentes de una Profesión (entorno de transmisión). Serie materiales de 
cátedra: Secretaría de Cultura y Comunicación, Facultad de Psicología, Universidad de Buenos Aires; Rossi, L. (2001). Psicología Pre-profesional en Argentina: Impacto de los cambios demográficos y políticos en las condiciones de surgimiento y estabilidad de las instituciones aplicativas. Revista del Instituto de Investigaciones, 6(2).; Talak, A. M. (2000). Los primeros desarrollos académicos de la psicología en la Argentina. Actas del Primer Encuentro Argentino de Historia de la Psiquiatría, la Psicología y el Psicoanálisis. Buenos Aires, Argentina; Vezzetti, H. (2004). Los comienzos de la psicología como disciplina universitaria y profesional. In F. Neiburg $\&$ M. Plotkin (Eds.), Intelectuales y expertos. La constitución del conocimiento social en la Argentina (pp. 293-326). Buenos Aires: Paidós; Vezzetti, H. (1996). Los estudios históricos de la psicología en la Argentina. Cuadernos Argentinos de Historia de la Psicología, (2), 79-94.

${ }^{3}$ Directora en Proyecto de Investigación "Perspectivas de género en el abordaje de la historia de la psicología y el psicoanálisis en Argentina. El rol de la mujer en el período 1900-1960", Facultad de Psicología, Universidad de Ciencias Empresariales y Sociales (U.C.E.S), 2011-2015. Directora Proyecto de Investigación “Género, historia y subjetividad. Las mujeres y pre-profesión de la psicología (1900-1950) desde la perspectiva de las representaciones sociales", Facultad de Psicología, Universidad de Ciencias Empresariales y Sociales (U.C.E.S) y continúa.

4 Para una ampliación de temas tratados se remite a los siguientes artículos: Falcone (2011). La mujer y sus estereotipos retratados en artículos y anuncios publicitarios de la década del treinta". Actas del XII Encuentro Argentino de Historia de la Psicología, la Psiquiatría y el Psicoanálisis (pp. 35-46). Buenos Aires, Argentina, 12. ISSN 1851-4812. Falcone (2009). Representaciones de género y subjetividad en las mujeres. Análisis de los discursos dominantes en la década del '30 en Argentina, XXXII Congreso Interamericano de Psicología de la Sociedad Interamericana de Psicología (SIP). Falcone (2008, Agosto 7-9). Historias de Género. Las mujeres y sus roles en la primera mitad del s. XX en Argentina. Simposio sobre Género e Historia, XIV Jornadas de Investigación y Cuarto Encuentro de Investigadores en Psicología del Mercosur. Facultad de Psicología, U.B.A. Falcone (2006, Octubre 3-4). Moda, género y subjetividad en las primeras décadas del siglo XX en Argentina. Mesa Redonda Género e Historia Psi. IX Encuentro Argentino de historia de la Psicología, la Psiquiatría y el Psicoanálisis, Bs.As., Buenos Aires, Argentina, 9.

${ }^{5}$ Véase para una ampliación: Carpintero, E., \& Vainer, A. (2004). Las huellas de la memoria. Psicoanálisis y Salud Mental en la Argentina de los 60 y 70 (1957-1969). Buenos Aires: Topia, entre otros y nuestros propios artículos científicos: entre otros, Falcone (2004). Psicología en Argentina en el periodo pre-profesional. La línea asistencial: formación en psicodiagnóstico y psicoterapia; Falcone (2004), XI Anuario de Investigaciones, Facultad de Psicología, U.B.A., 495- 503.

Financiamiento: Universidad de Buenos Aires. Argentina. Proyecto Ubacyt. Ciencia Y Tecnica.

Este artigo de revista Estudos e Pesquisas em Psicologia é licenciado sob uma Licença Creative Commons Atribuição-Não Comercial 3.0 Não Adaptada. 